a dwarf in comparison with those seen by $\mathrm{Mr}$. Munro and $\mathrm{Mr}$. Quin. Doubtless the broken-down and pulverised corallines fill up many a crack in the reef's limestone. Should Mr. Munro be desirous of seeing some of his old West Indian corallines, I shall be glad to show him some microscopical results of work upon them.

Geological Society, May I

P. Martin Duncan

\section{History of Magnetism}

A PARAGRAPH in the article on "The Early History of Mag. netism," in your last number, contains a passage which requires, I think, a note of explanation. The writer says: "A Latin letter ascribed to Peter Adsiger, 1269, preserved among the manuscripts of the University of Leyden, contains the following remark on the declination of the needle ..." Now Humboldt, on the authority of Libri, denies the existence of the passage in the Leyden MSS., affirming that it is only an interpolation in a Paris copy. But what is of more importance, he also states that the title of the letter is "Epistola Pêtri P. de Maricourt ad Sigernum de Foucoucourt." E. Walker, in his well-known essay on Magnetism, refers to Cavallo as quoting the supposed letter of Adsiger.

S. J. PERRY

\section{Meteorological Society}

WHILE thanking you for your friendly notice of the Annual Report of this Society, I trust you will allow me to state that we have not made "the mistake in science regarding the height of the thermometers above the ground," as very naturally imagined by you from the matter not having been mentioned. The fact is, we have been unusually strict on that point ; our thermometers are all 4 feet (writhin, perhaps, 2 in. + or - ), and as the uniformity was so strict, it was considered useless to repeat the statement for each station, and so, finally, it escaped mention altogether in the printed abstract. Of course the question (Report, p. 52), "What is the height of the bulbs above grass?" is duly answered on the MS. inspection forms deposited in the library of the Society.

May I, in conclusion, express the hope that the example which we have set by publishing the lithograph ground-plans, and which you so highly approve, may be generally followed both in this country and abroad?

Meteorological Society, 30, Great George Street,

Westminster, S. W., April 28

\section{Destruction of Flowers by Birds}

THE enclosed blossoms of the common "wild" cherry (Prunus avium, L.) have been mutilated in a precisely similar mamer with those of the blackthorn noticed about a year ago in NATURE (vol. xii. p. 26), the petals and stamens still adhering to the separated limb of the calyx, which has been cut through at the exact level of the ovary, which has perhaps been the object of attack. Orchard trees in the neighbourhood from the same stock have also suffered to a serious extent, but the wallcherries $(P$. cerasus, $L$.), which are later in flowering, have hitherto been untouched.

R. A. PRYOR

Hatfield, May 2

\section{OUR ASTRONOMICAL COLUMN}

The Nebula in ORION.-M. Tisserand, Director of the Observatory at Toulouse, commenced on Feb. I7 of the present year, a close examination of the small stars in the vicinity of the trapezium in the great nebula of Orion, with the Foucault telescope of $\mathrm{O}^{\mathrm{m} \cdot 80}$ aperture, which had been completely mounted at the beginning of the same month. To facilitate the study of this region, which it is intended shall form part of the work with this fine instrument, a chart was prepared on a large scale containing the 155 stars, the positions of which relatively to $\theta^{1}$ Orionis, were determined by M. O. Struve Cobservations de la Grande Nebuleuse d'Orion in the St. Petersburg Memoirs, vol. v.); of these 155 stars it may be mentioned that I 50 occur in Sir John Herschel's list in the volume of observations made at the Cape of Good Hope. Especial attention was directed at Toulouse during the few weeks that the nebula could be observed in the last season, to the stars which M. O. Struve had indicated as variable. The star $\Pi\left(\Delta a \ldots-7^{\prime \prime} \cdot 3, \Delta \delta \ldots-27^{\prime \prime} \cdot 6\right)$ which is not in Herschel's catalogue, was noted on Feb. I7 and 21 at the extreme limit of visibility : on following days, when the sky was more transparent, it could not be discerned; at maximum according to Struve this star is of the twelfth magnitude, the smallest star which can be distinctly seen in the Pulkowa refractor being considered 13.5 - a very different scale of magnitude, it will be remarked, from that of Bessel ; No. $78\left(\Delta a \ldots+34^{\prime \prime} 5\right.$, $\left.\Delta \delta \ldots+9^{\prime \prime} \cdot 7\right)$, varying, according to Struve, from $12 \cdot 5$ to invisibility, was not discerned ; No. $75\left(\Delta a \ldots+2 r^{\prime \prime} \cdot 3\right.$, $\left.\Delta \delta \ldots+39^{\prime \prime} \cdot 2\right)$ was $14-15$ on March 14; Tisserand found No. V. of the Pulkowa list $\left(\Delta a \ldots+378^{\prime \prime} \cdot 3\right.$, $\Delta \delta \ldots+66^{\prime \prime} 3$ ) extremely faint on Feb. 24, and quite invisible subsequently, whence he concludes this star to be also variable, and that its non-insertion by Herschel may have arisen from its being at a minimum at the epoch of his observations.

Thirty-two stars have been remarked at Toulouse, which are not in the Pulkowa catalogue; of these fifteen occur in Bond's catalogue, in vol. v. of "Annals of the Harvard Observatory"; the remaining seventeen which have not, as it appears, been previously observed, are generally very faint, the only notable exceptions being in the cases of two stars, which have the following estimated co-ordinates relative to $\theta^{1}$.

$$
\begin{array}{ccccccccc}
\Delta \alpha & \ldots & \ldots & +180^{\prime \prime} & \ldots & \Delta \delta & \ldots & \ldots & -180^{\prime \prime} \\
, & \ldots & \ldots & -\mathrm{IIO}^{\prime \prime} & \ldots & , & \ldots & \ldots & -480^{\prime \prime}
\end{array}
$$

The first star was 13 (an object termed très belle with the Toulouse instrument) on February 17 , but had become extremely faint on March 14 and 26 . The second star is estimated 13, almost as bright as its neighbour, No. 55 of Struve's catalogue. M. Tisserand states that he has not been able to recognise all the stars in Bond's catalogue, more patticularly in the neighbourhood of the trapezium.

The numerous variable stars, which we have now reason to suppose exist in the nebula of Orion, certainly form one of the most significant and interesting features in the history of that grand object.

It may be added here that M. Tisserand has also employed the powerful optical means now at his command, upon observations of the satellites of Uranus.

New Minor Planets. - Still another small planet is announced during the last week. It was found by $M$. Perrotin at Toulouse on April 26, in R.A. 14h. I Im. 485, N.P.D. $96^{\circ} 24^{\prime}$; twelfth magnitude.

The planet detected by Prof. Watson at Ann Arbor on April I9 is called No. 16I in the Astronomische Nach. richten. These numbers, however, are now in much confusion, and names for those which are observed a sufficient length of time to allow of the determinacion of elements have an obvious advantage over the system of leaving these planets to be distinguished by a number only. As regards numbers there is even doubt as far back as No. 149, which has not yet been shown to be distinct from Frigga (No. 77).

BIELA'S COMET AND THE NOVEMBER METEOR-STREAM. -If we take for the orbit of the November meteor-stream the elements calculated by Prof. J. C. Adams, and communicated to the Royal Astronomical Society in April, 1867 , and for Biela's comet a mean of the sets of elements for the two nuclei in 1866, given by Clausen in "Melanges Niathématiques et Astronomiques," \&c., t. iii., of the Imperial Academy of St. Petersburg, we find for the least distance between the tracks of the comet and the meteors, 0 '054, the mean distance of the earth from the sun being taken as unity. This nearest point of approach is in heliocentric longitude $6 \mathrm{I}^{\circ} 3 \mathrm{O}^{\prime}$ (equinox of 1866), where we have-

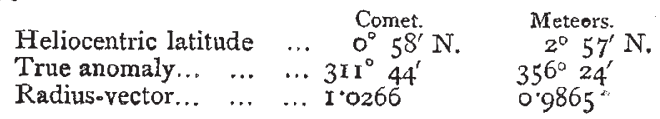

